\title{
A Highly Quantitative Multi-Well Format Assay for Studying the Effect of Extracellular Matrix Mechanics on the Bacterial Infection of Endothelial Cells
}

\author{
By Effie Bastounis* \\ Julie A. Theriot ${ }^{\dagger}$
}

Bacterial pathogens can cause systemic infections by disseminating from the initial focus of infection to distant organs through the blood vasculature. To colonize tissues from the bloodstream, many bacteria need to adhere to endothelial cells (ECs) that line the vessels' inner lumen, invade them and then spread from cell to cell. ECs are highly mechanosensitive and respond to mechanical cues, including the stiffness of the matrix on which they adhere, by dynamically re-arranging their cytoskeleton, cell-cell adhesions, and adhesions to the extracellular matrix. We hypothesize that bacterial infection of ECs might also be affected by the mechanics of the host ECs, the latter being influenced by the stiffness of the matrix on which ECs reside. To test this hypothesis, we developed a novel multi-well format assay that allows assessing in a highly quantitative manner the differential effect of matrix stiffness on EC bacterial infection. We demonstrate that, using our assay, we can quantify infection efficiency through flow cytometry and microscopy, and also measure the effects of infection on EC mechanics through traction force microscopy. Our method allows for the analysis of the effect of tissue-relevant mechanics on EC infection, which is a critical step towards understanding the biomechanical interactions between ECs, their extracellular matrix and pathogenic bacteria.

Keywords: Bacterial infection, Endothelial cells, Flow cytometry, Polyacrylamide hydrogels, Traction force microscopy.

\section{Introduction}

The endothelial cell (EC) monolayer of the vascular wall is a major target for pathogenic bacteria to systemically disseminate (Lemichez et al., 2010). ECs are highly mechanosensitive and respond to varying mechanical cues, including the stiffness of their extracellular matrix (ECM), by altering both their gene expression profiles and the organization of their cytoskeletons and adhesion architecture. These mechanosensitive responses in turn affect the regulation of EC mechanics and motility (Ingber, 2008; Yeh et al., 2012; Hur et al., 2012; Santos et al., 2015; Collins et al., 2014). Although EC mechanics and response to ECM stiffness have been studied extensively, the relationship

\footnotetext{
* Postdoctoral Scientist, Stanford University, USA.

${ }^{\dagger}$ Professor, Stanford University, USA.
} 
between bacterial infection and the biomechanics of the endothelium is largely unknown.

Several bacterial species, including the Gram-positive facultative pathogen Listeria monocytogenes (L.m.) utilize the actin cytoskeleton of host cells to invade into their cytoplasm and later spread to neighboring cells (Cossart and Kocks, 1994; Tilney and Portnoy, 1989). They do so by expressing proteins capable of exploiting various actin polymerization pathways to produce actin tails, which allow them to propel forward (Dabiri et al., 1990; Theriot et al., 1992; Kocks et al., 1992). As a result of infection, the hosts' actin cytoskeleton needs to dynamically rearrange in a manner that depends on the infecting species (Gouin et al., 1999; Choe and Welch, 2016). L.m. is a ubiquitous foodborne bacterium that can move from the intestinal epithelium to distant organs protected by different types of vascular endothelium to cause meningitis and encephalitis when it crosses the blood-brain barrier and to cause spontaneous abortion when it crosses the placenta (Vazquez-Boland et al., 2001; Jackson et al., 2010). It uses multiple pathogenic strategies to infect host cells, however little is known about how this bacterium can access, infect and bypass the endothelium and what is the effect of the mechanical heterogeneity of vessels in regulating its invasion and spread (Drevets et al., 1995; Wilson and Drevets, 1998).

Traditionally bacterial infection has been studied in tissue culture using glass or polystyrene surfaces, both of which are too stiff to be physiologically relevant (Rajabian et al., 2009; Vazquez-Boland et al., 2001; Dussurget et al., 2004). To be able to recapitulate in vivo conditions and to determine in a systematic way the role of the ECs' matrix stiffness on the efficiency of bacterial infection, we developed a novel assay based on manufacturing thin microbead-embedded polyacrylamide hydrogels of varying stiffness on 24-well glass-bottom plates (Ahmed, 2015; Mih et al., 2011; Georges et al., 2006). ECs were seeded on the hydrogels and subsequently infected with bacterial strains that become fluorescent several hours after internalization (Zeldovich et al., 2011). Percentage of infection as a function ECM stiffness was assessed through flow cytometry. Flow cytometry results were also confirmed through immunostaining and subsequent automated fluorescence microscopy. Finally since our hydrogels are thin enough and embedded with fluorescent beads one can also perform Traction Force Microscopy (TFM) to assess the effect of infection on the traction stresses that infected ECs exert on their matrices during the course of infection. Our assay can be applied to study the role of the ECM environment on infection using different cell lines or pathogens. 


\section{Experimental Procedures}

Fabrication of Thin Two-layered Polyacrylamide Hydrogels on 24-well Glassbottom Dishes

24-well glass bottom plates (MatTek) were incubated for $1 \mathrm{~h}$ with $500 \mu \mathrm{l}$ of $2 \mathrm{M} \mathrm{NaOH}$. Wells were rinsed with distilled water and then $500 \mu \mathrm{l}$ of $2 \%$ (3Aminopropyl)triethoxysilane (Sigma) in $95 \%$ ethanol were added in each well for $5 \mathrm{~min}$. Wells were rinsed again with water and $500 \mu \mathrm{l}$ of $0.5 \%$ gluteraldehyde were added in each well for $30 \mathrm{~min}$. Wells were rinsed with water and dried at $60^{\circ} \mathrm{C}$.

Polyacrylamide hydrogel fabrication was done as previously described with a few modifications for the specific experiments (Bastounis et al., 2014; Georges et al., 2006; Vincent et al., 2013). To prepare hydrogels of varying stiffness, mixtures were prepared that contained 3-10\% acrylamide and $0.06-$ $0.6 \%$ bis-acrylamide. Specifically, $0.6 \mathrm{kPa}$ hydrogels contained $3 \%$ acrylamide and $0.06 \%$ bis-acrylamide, $3 \mathrm{kPa}$ hydrogels contained $5 \%$ acrylamide and $0.1 \%$ bis-acrylamide, $10 \mathrm{kPa}$ hydrogels contained $10 \%$ acrylamide and $0.1 \%$ bisacrylamide, $20 \mathrm{kPa}$ hydrogels contained $8 \%$ acrylamide and $0.26 \%$ bisacrylamide, $70 \mathrm{kPa}$ hydrogels contained $10 \%$ acrylamide and $0.6 \%$ bisacrylamide. For each stiffness two mixtures were prepared the second of which contained $0.03 \% \quad 0.1 \mu \mathrm{m}$ diameter fluorescent beads (Fluorospheres, Invitrogen). Mixtures were then degassed for $15 \mathrm{~min}$ to remove oxygen from the solutions that prohibits polymerization.

First $0.06 \%$ APS and $0.43 \%$ TEMED were added to the solutions containing no beads to initiate polymerization and then $3.6 \mu \mathrm{l}$ of the mixture was added at the center of each well, capped with $12 \mathrm{~mm}$ untreated circular coverslips and allowed to polymerize for $20 \mathrm{~min}$. The coverslips were then lifted via a syringe needle with a small hook at its tip and then $2.4 \mu \mathrm{l}$ of the mixture containing the tracer beads was added, sandwiched again with a 12 $\mathrm{mm}$ untreated circular glass coverslip, gently pressed downwards by means of forceps and allowed to polymerize for $20 \mathrm{~min}$. 50mM HEPES $\mathrm{pH}=7.5$ was added in the wells and coverslips were removed using the syringe needle and forceps. Hydrogels were exposed to $\mathrm{UV}$ for $1 \mathrm{~h}$ to allow sterilization. Hydrogels were then activated by adding $200 \mu 10.5 \% \mathrm{w} / \mathrm{v}$ heterobifunctional crosslinker Sulfo-SANPAH (ProteoChem) in 1\% DMSO and 50mM HEPES $\mathrm{pH}=7.5$, on the upper surface of the hydrogels and exposing to UV light for $10 \mathrm{~min}$. After activation, the hydrogels were washed with 50mM HEPES pH = 7.5 to remove excess crosslinker and were coated with $200 \mu \mathrm{l}$ of $0.25 \mathrm{mg} / \mathrm{ml}$ rat tail Collagen I (Sigma-Aldrich) in 50mM HEPES overnight at room temperature. Hydrogel thickness was measured by locating the top and bottom planes of the hydrogel and subtracting the $\mathrm{z}$ positions. Prior to seeding cells, hydrogels were incubated with cell media to allow equilibration for $30 \mathrm{~min}$. 


\section{HMEC-1 Culture and Infection with Listeria Monocytogenes}

HMEC-1 cells (generous gift from the Matthew Welch and Rebecca Lamason, UC Berkeley) were maintained in MCDB 131 medium supplemented with $10 \%$ fetal bovine serum, $10 \mathrm{ng} / \mathrm{mL}$ epidermal growth factor, $1 \mu \mathrm{g} / \mathrm{mL}$ hydrocortisone and $2 \mathrm{mM}$ L-glutamine. HMEC-1s were added on the hydrogels 2 days prior to infection at a concentration of $2 \times 10^{5}$ cells/well to ensure confluency by day 2 .

L.m. strains were grown overnight, spinning, at $30^{\circ} \mathrm{C}$ in $\mathrm{BHI}$ media (Gibco) with $7.5 \mu \mathrm{g} / \mathrm{mL}$ chloramphenicol (CAM). Infections were performed in normal growth media lacking antibiotic. To synchronize invasion, L.m. were added on the HMEC-1 cells seeded on the hydrogel matrices and samples were spun for $10 \mathrm{~min}$ at $200 \mathrm{x}$ g prior to incubation. After $30 \mathrm{~min}$, samples were washed 4 times in PBS and after additional $30 \mathrm{~min}$, media was replaced with media supplemented with $20 \mu \mathrm{g} / \mathrm{mL}$ gentamicin. Multiplicity of infection (MOI) was determined by plating bacteria at different dilutions, on BHI agar plates with $7.5 \mu \mathrm{g} / \mathrm{mL}$ CAM and measuring the number of colonies formed.

\section{Immunostaining of Extracellular Adhering Bacteria}

HMEC-1 cells were infected with a L.m. strain that constitutively expresses GFP using centrifugation as described above. 20 min post-infection 1 $\mu \mathrm{l}$ of $1 \mathrm{mg} / \mathrm{ml}$ Hoechst dye was added in each well to stain the cells' nuclei. 30 min post-infection cells were washed 4 times in PBS and fixed in $4 \%$ paraformaldehyde in PBS for $10 \mathrm{~min}$ at room temperature. Following a wash with PBS samples were incubated with $100 \mu \mathrm{l}$ of $1 \%$ BSA in PBS with antiL.m. primary antibody ab35132 (Abcam) in 1:100 dilution for $30 \mathrm{~min}$. Samples were washed in PBS 3 times and then incubated with $100 \mu$ of $1 \%$ BSA in $1 x$ PBS Alexa Fluor 546 goat anti-rabbit secondary antibody (Invitrogen) in 1:250 dilution. Samples were washed 3 times in PBS and stored in $1 \mathrm{ml}$ PBS for imaging. Imaging was performed using a 40X Plan Fluor NA 0.60 objective. 500-1000 cells were analyzed per condition.

\section{Flow Cytometry of HMEC-1 Cells Infected with Listeria Monocytogenes}

$8 \mathrm{~h}$ post-infection infected HMEC-1 cells were detached from the hydrogel using a mixture of $200 \mu \mathrm{l}$ of $0.25 \%$ trypsin-EDTA and $0.05 \%$ Collagenase (Sigma) with which the cells were incubated for $10 \mathrm{~min}$. Solutions in each sample were pipetted up and down for 6 times to ensure single cell suspensions and $200 \mu 1$ of complete media was added to inactivate trypsin. Solutions were transferred into $5 \mathrm{ml}$ polystyrene tubes with a $35 \mu \mathrm{m}$ cell strainer cap (Falcon) and then samples were immediately analyzed by flow cytometry on the Scanford FACScan analyzer (Custom Stanford and Cytek upgraded FACScan). 10,000-20,000 cells were analyzed per replicate. 


\section{Traction Force Microscopy of EC Monolayers}

Multi-channel time-lapse sequences of fluorescence (to image the beads and the bacteria) and phase contrast images (to image the cells) were acquired using an inverted Nikon Diaphot 200 with a CCD camera (Andor Technologies) using a 40X Plan Fluor NA 0.60 objective and the MicroManager software package (Edelstein et al., 2014). The microscope was surrounded by a cage incubator (Haison) maintained at $37^{\circ} \mathrm{C}$ and $5 \% \mathrm{CO}_{2}$. Images were acquired every $10 \mathrm{~min}$ for 4 to $12 \mathrm{~h}$. Subsequently, at each time interval we measured the 2D deformation of the substrate at each point using a technique similar to particle image velocimetry. We calculated the local deformation vector by performing image correlation between each image and an unstretched reference image which we acquired by adding 10\% SDS at the end of each recording to detach the cells from the hydrogels. We used interrogation windows of $32 \times 16$ pixels. The $2 \mathrm{D}$ traction stresses that cells exerted on the hydrogel were calculated as described elsewhere (del Álamo et al., 2007; Hur et al., 2012).

\section{Results}

Fabrication of Thin 2-layered Polyacrylamide Hydrogels of Tunable Stiffness with Embedded Fluorescent Beads on 24-well Glass Bottom Plates

24-well glass bottom plates were treated appropriately to allow subsequent attachment of polyacrylamide hydrogels (see first subsection of Experimental Procedures, Figure 1 A). Two-layered thin polyacrylamide hydrogels, the upper layer of which contained $0.03 \%$ carboxylate-modified yellow latex beads $0.1 \mu \mathrm{m}$ in diameter were built sequentially on top of the glass bottoms (Figure 1 B-F). To prepare matrices of different physiologically relevant stiffnesses, mixtures were prepared that contained $3-10 \%$ acrylamide and $0.06-0.6 \%$ bisacrylamide which allowed us to fabricate hydrogels with stiffness ranging from $0.6 \mathrm{kPa}$ to $70 \mathrm{kPa}$. Six replicates for each hydrogel stiffness were fabricated to assess reproducibility of the results. Hydrogels were sterilized under UV and then activated via Sulfo-SANPAH to enable coating with collagen I and therefore allow cell attachment (Figure 1 G-I). The thickness of the hydrogels was measured through microscopy and was on average $40 \mu \mathrm{m}$ but varied slightly with stiffness. Hydrogels were seeded with ECs (Figure $1 \mathrm{~J}-\mathrm{K}$ ) and bacterial infection was performed using centrifugation to synchronize bacterial invasion (Figure $1 \mathrm{~L}-\mathrm{M}$ ). 
Figure 1. Flow Cytometry Assay to Measure Bacterial Infection of Cells Residing on Polyacrylamide (PA) Hydrogels of Tunable Stiffness on 24-well Plates. A. Glass coverslips of wells are treated appropriately to allow hydrogel attachment. B. $3.6 \mu$ l of PA solution with no beads is deposited on the glass bottoms. C. Solution is covered by $12 \mathrm{~mm}$ untreated circular coverslip to allow polymerization. $\boldsymbol{D}$. After $20 \mathrm{~min}$ coverslip is removed by means of a needle syringe with a hook at its tip. E. $2.4 \mu \mathrm{l}$ of PA solution with tracer microbeads is deposited on top of the bottom layer, sandwiched with a $12 \mathrm{~mm}$ untreated circular glass coverslip and gently pressed downwards with forceps. F. After 20 min, HEPES is added in the well and coverslip is removed by means of a needle syringe. G. 24-well plate is placed under $U V$ for $1 h$ to ensure sterilization. $\boldsymbol{H}$. Sulfo-SANPAH containing solution is added on the hydrogels, which are then placed under UV for $10 \mathrm{~min}$. I. Hydrogels are washed in HEPES and then incubated with collagen I in HEPES overnight. J. Hydrogel is incubated with cell culture media for 30 min to ensure equilibration. $\mathbf{K}$. Cells are seeded and incubated for 48 h. L. Bacteria are added and cell infection is synchronized by centrifugation. M. 1 h post-infection bacteria are washed 4 times in PBS and media supplemented with antibiotic is added. N. Bacteria start fluorescing at approximately $4 \mathrm{~h}$ post-infection. $\boldsymbol{O}$. Cells are detached from their matrix using a mixture of $0.25 \%$ trypsin-EDTA and collagenase and solutions are transferred to polystyrene tubes with cell-strainer caps for flow cytometry
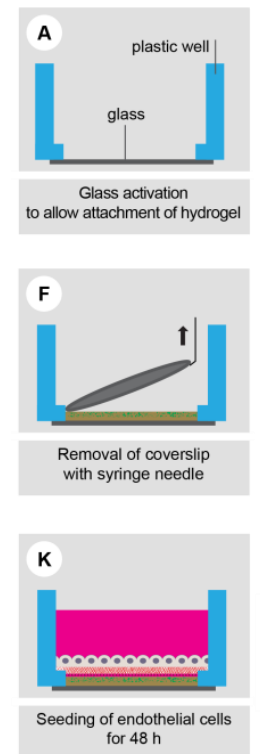

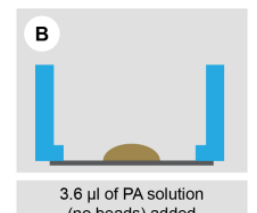

$3.6 \mu$ l of PA solution
(no beads) added
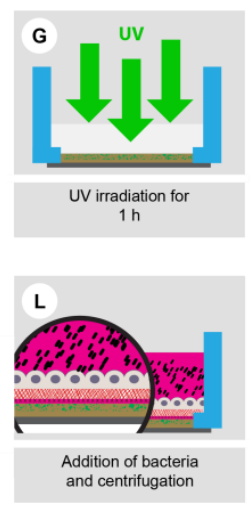
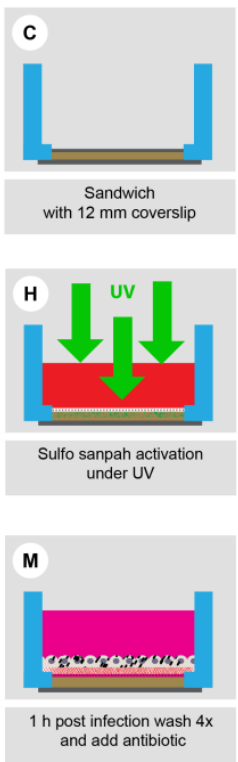

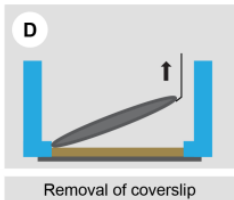

Removal of coverslip
with syringe needle
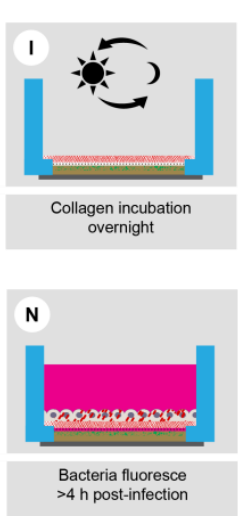
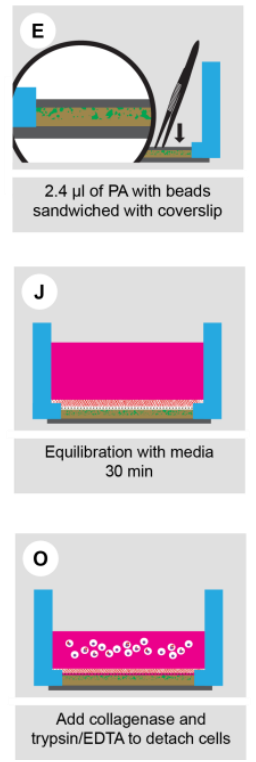

Flow Cytometry of ECs Infected with Fluorescently Labeled Bacteria Residing on Matrices of Varying Stiffness

We sought to evaluate what effect the ECM stiffness would have on L.m. infection of EC hosts. For that, we manufactured polyacrylamide hydrogels of varying physiologically relevant stiffnesses ranging from 0.6 to $70 \mathrm{kPa}$ on $24-$ well plates with 6 replicates per condition as described above (Bastounis et al., 
2014; Georges et al., 2006; Vincent et al., 2013; Birukova et al., 2013; Mih et al., 2011) (Figure 1 A-I). We then seeded ECs at a concentration of $2 \times 10^{5}$ cells per well for $48 \mathrm{~h}$ (Figure $1 \mathrm{~K}$ ). Subsequently we infected the host ECs with a L.m. strain that expresses a fluorescent marker several hours after internalization (actAp::mTagRFP) (Zeldovich et al., 2011), allowing the detection only of intracellular bacteria (Fig. $1 \mathrm{~L}-\mathrm{N}$ ). $8 \mathrm{~h}$ post-infection we detached the infected ECs from the hydrogels using a mixture of $0.25 \%$ trypsin-EDTA and collagenase and transferred the solutions in polystyrene tubes with cell strainer caps to ensure single EC suspensions (Figure $1 \mathrm{O}$ ). Samples were then analyzed through flow cytometry and percentage of infected ECs per well was quantified. We selected (gated) the bulk of the distribution of cell counts using the forward scatter area versus side scatter area plot to ensure we analyzed single cells and not debris or doublets or triplets of cells (Figure 2 A). Since uninfected cells exhibit autofluorescence, we always measured fluorescence of control uninfected cells (see beige plot in Fig. 2 B-C) and then gated our population of infected cells making sure we excluded all cells that lie where the uninfected sample lies (Figure 2 B-C). Preliminary results depicted in Figure 2 show that EC infection with L.m. is more efficient for ECs residing on stiffer matrices (Figure $2 \mathrm{~B}-\mathrm{D}$ ).

Figure 2. L.m. Infection of ECs Residing on Hydrogels of Varying Stiffness. Human microvascular endothelial cells (HMEC-1s) were infected with L.m.(actAp::mTagRFP) and infection was analyzed by flow cytometry. A. Side scatter versus forward scatter plot for a representative population of HMEC-1s coming from a single well. Gating was performed to select the bulky distribution of cells and exclude debris (left) and doublets or triplets of cells (right). $67.8 \%$ of the total counts were selected. B-C. Histograms of L.m. fluorescence intensity per cell, for HMEC-1s plated on soft $0.6 \mathrm{kPa}(\mathrm{B})$ and stiff $70 \mathrm{kPa}$ hydrogels $(C)$. Histograms for 4-6 replicates are shown in different colors. Control uninfected cells' histogram is shown in beige. Based on the autofluorescence of the control group a gate was defined (see black line) showing what is considered uninfected (left) and infected (right). Multiplicity of infection (MOI) is 100 bacteria/cell and cells are examined $8 \mathrm{~h}$ postinfection. D. Boxplots of percentage of infected HMEC-1s versus hydrogel stiffness. Circles represent outliers, and the boxplots' notched sections show the 95\% confidence interval around the median. Asterisks denote statistically significant differences $(* *: p<0.01, *: p<0.05$ )
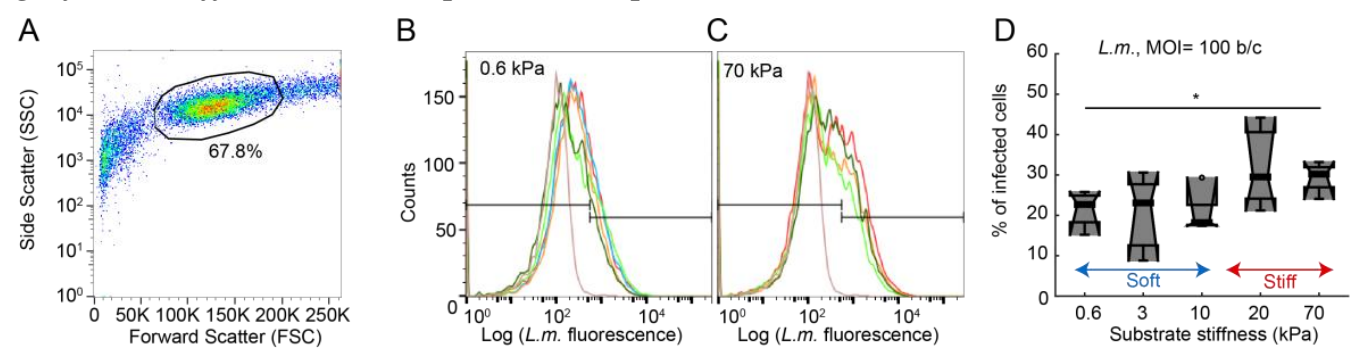
Immunostaining of Constitutively Fluorescent L.m. Infecting ECs Residing on Varying Stiffness Hydrogels and Quantitation of Infection through Automated Fluorescence imaging

To ensure that density of ECs residing on different stiffness hydrogels is similar, and to be able to differentiate between bacteria that are adhering on ECs versus the ones that have been successfully internalized by ECs, we performed immunostaining followed by microscopy. Briefly, ECs residing on varying stiffness hydrogels were infected with constitutively GFP fluorescent L.m. (Figure 3 B, E). 30 min post-infection bacteria were washed away (see third subsection in Experimental Procedures), ECs' nuclei were stained with Hoechst and samples were fixed with formaldehyde. Samples were then incubated with an anti-L.m. primary antibody and subsequently with an RFP secondary antibody to enable visualization of the adhering (extracellular) bacteria only (Figure 3 C, F). Samples were stored in PBS for imaging. We used fluorescence microscopy and the HCS generator plugin of the MicroManager software (Edelstein et al., 2014) to automatically acquire multiple images across wells of the ECs (phase image), the cell nuclei (blue), the total number of bacteria (green) and the bacteria adhering on cells only (red) (Figure 3 A-F). We then used MATLAB and an automated image analysis script (Selinummi et al., 2005) to quantify in each field of view the number of nuclei, the number of adhering L.m. and the number of internalized L.m. (Figure 3 L, $\mathrm{G}, \mathrm{J})$. The above parameters were also normalized to the number of cells in the field of view (Figure $3 \mathrm{H}, \mathrm{K}$ ) to ensure that the differences we captured were not due to varying cell density since differences in proliferation rates have been suggested to occur when cells are grown on different stiffness matrices (Yeh et al., 2012). Our preliminary results suggest that adhesion is increased for ECs residing on stiff hydrogels but that invasion efficiency (bacteria internalized/total number of bacteria) is similar irrespective of substrate stiffness (Figure 3 G-I). 
Figure 3. Outside Immunostaining of Constitutively Fluorescent L.m. Infecting ECs Residing on Varying Stiffness Hydrogels. A. Representative example of cell nuclei (blue channel) superimposed on the phase image of infected with L. monocytogenes HMEC-1s residing on a soft $3 \mathrm{kPa}$ hydrogel. Cells were fixed 30 minutes post-infection. Multiplicity of infection is 7.5 bacteria per cell. Scale bar is $23 \mu \mathrm{m}$. B. Image of all the bacteria (outside and inside, green channel) corresponding to the image shown in panel A. C. Image of the adhering outside bacteria (red channel) corresponding to the image shown in panel A. Arrows indicate bacteria that have been internalized and are therefore only shown on the green but not the red channel. D-E. Same as panels A-C but for infected with L. monocytogenes HMEC-1s residing on a stiff $70 \mathrm{kPa}$ hydrogel. $\mathbf{G}-\mathbf{L}$. Boxplots referring to $N=20$ images (2 replicates per condition) captured for infected $\mathrm{HMEC}$-1s residing on soft $3 \mathrm{kPa}(\mathrm{red})$ and stiff $70 \mathrm{kPa}$ (red) hydrogels. Boxplots show the distribution of the: $\boldsymbol{G}$. total number of bacteria; $\boldsymbol{H}$. total number of bacteria normalized with the number of nuclei; I. invasion efficiency determined as the ratio of the internalized bacteria divided to the total number of bacteria; J. internalized bacteria; I. internalized bacteria normalized with the number of nuclei; I. number of nuclei. Circles represent outliers, and the boxplots' notched sections show the $95 \%$ confidence interval around the median. Asterisks denote statistically significant differences $(* *: p<0.01, *: p<0.05)$
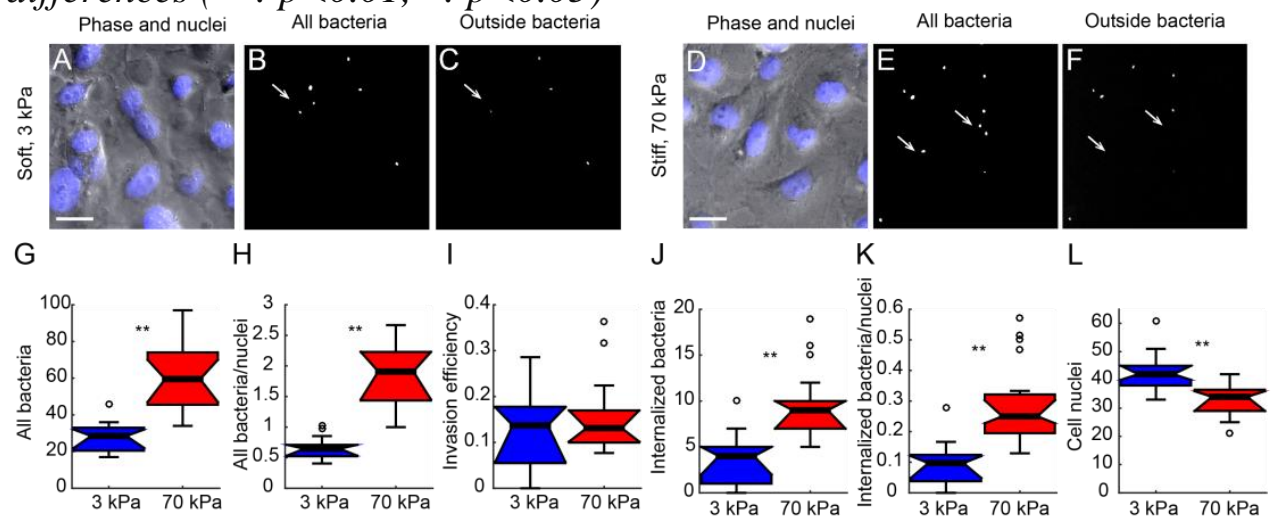

Traction Force Microscopy of Multiple Conditions Simultaneously

Flow cytometry and differential immunostaining of ECs infected with L.m. allows assessment of the effect of ECM mechanics and particularly stiffness on the bacterial infection of ECs. To turn the problem upside down and examine the effect of infection on the biomechanics of the endothelium, we can compute the traction stresses that ECs exert during the course of infection on compliant hydrogels. Our hydrogels are thin enough so that cells and fluorescent microbeads embedded in the hydrogels' upper surface can be well imaged using a long working distance $40 \mathrm{X}$ NA=0.6 objective. In addition the upper bead layer of the hydrogels is extremely thin confining the beads in one focal plane and ensuring no out of focus light. This feature enables the performance of TFM measurements to calculate the traction stresses that infected ECs exert on their matrix (del Álamo et al., 2007; Hur et al., 2012). In 
TFM, cells are seeded on the fluorescent micro-bead embedded elastic hydrogels of known mechanical properties (Figure 4 A and D). As cells crawl on the hydrogel, they induce deformations on it that can be tracked through the displacements of the beads (Alexander et al., 1992) (Figure 4 B and E). Solving the elastostatic equation in Fourier space enables the calculation of the traction stresses exerted by the cells on their matrix (del Álamo et al., 2007; Bastounis et al., 2011) (Figure $4 \mathrm{C}$ and F). Figure 4 depicts the deformation and traction stress maps of ECs residing on a $20 \mathrm{kPa}$ matrix at different time points postinfection. Panels A-C refer to uninfected control ECs whereas panels D-E refer to ECs infected with L.m. with a multiplicity of infection of 300 bacteria/cell. The outcome of this experiment suggests that infected ECs reduce over time the magnitude of the traction stresses they exert on their matrix.

Figure 4. Infected HMEC-1s Decrease their Traction Forces during the Course of Infection. A. Phase image (A), deformation field (B) and traction stress field $(C)$ of uninfected HMEC-1s residing on a $20 \mathrm{kPa}$ hydrogel. Colorbars of the deformation maps $(\mu \mathrm{m})$ and of the traction stress maps $(\mathrm{Pa})$ are shown on the right side of the heat maps. Images refer to three representative instants of time corresponding to 3, 7 and 21 h post-infection. (D-F) Same as panels A-C but for HMEC-1s infected with L.m. $($ MOI = 300 bacteria/cell $)$. Note that the images of the bacteria (red channel) are superimposed on the phase images of the HMEC1s. All traction force microscopy recordings were conducted simultaneously by imaging multiple wells using a 24-well plate where hydrogels were manufactured. Cell density during infection was $8 \times 10^{5}$ cells per well. Scale bar is $32 \mu m$
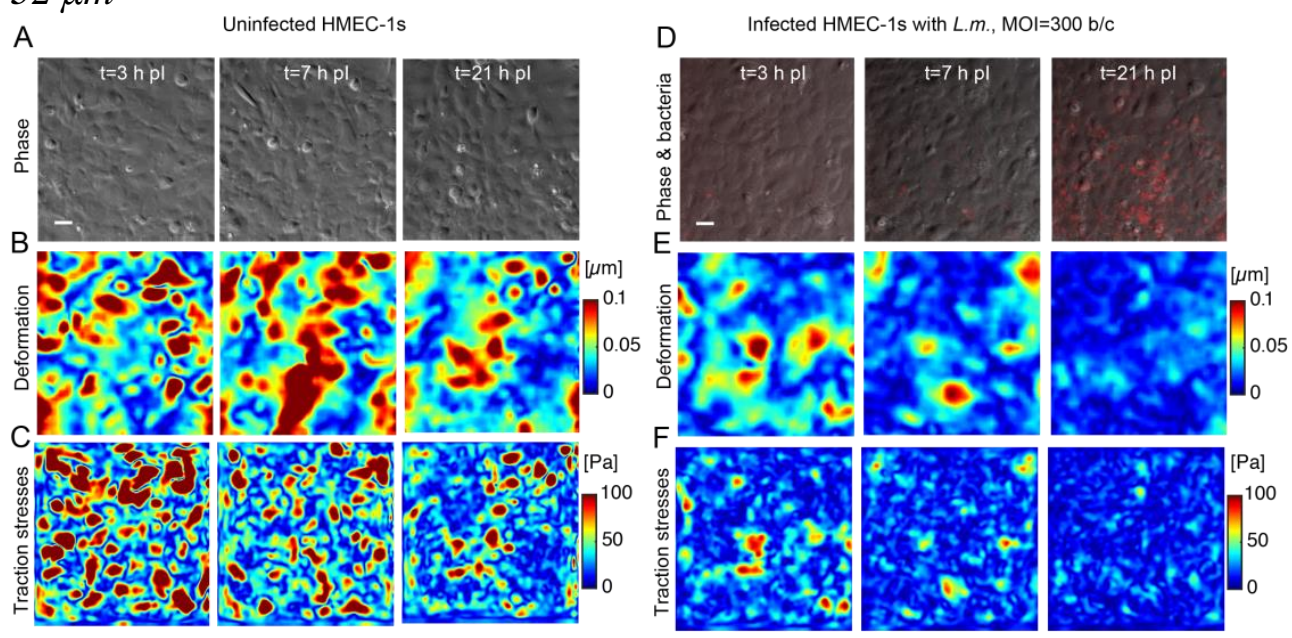

\section{Discussion}

The physical environment of cells including the stiffness of their extracellular matrix (ECM) is known to affect cellular motility, differentiation, proliferation and ultimately cell fate (Yeh et al., 2012; Ali et al., 2014; Tse and Engler, 2011; Chaudhuri et al., 2015; Minaisah et al., 2016). Although there 
have been many recent advances in understanding the complex biomechanical interaction between cells and their ECM, little is known about how bacterial infection of host cells is affected by the mechanics of the environment on which the hosts reside. To facilitate such studies, we developed a novel assay based on the well-established fabrication of polyacrylamide (PA) hydrogels of tunable stiffness on mutli-well glass bottom plates (Mih et al., 2011; Bastounis et al., 2014). Traditionally bacterial infection of host cells in tissue culture has been examined on glass or polystyrene surfaces. Our assay opens new highways by enabling the study of the interaction of bacteria with host cells in a much more physiological environment, since the stiffness of the hydrogel where ECs reside is comparable to the ECM stiffness were ECs naturally reside.

The novelty of our approach compared to previous techniques of PA fabrication (Bastounis et al., 2014; Mai et al., 2007; Mih et al., 2011) lies in three distinct features incorporated in our assay. First, hydrogels are manufactured on multi-well plates with glass bottoms allowing the examination of multiple conditions simultaneously which is crucial given that the outcome of such experiments can be influenced by many factors, including cell passage (generation), exact number of infecting bacteria, or seeding density. Second, our hydrogels are thin enough $(\sim 40 \mu \mathrm{m})$ to allow the performance of cell fixation and bacterial immunostaining followed by quantitation through automated microscopy and image processing. Thick specimens are often hard to image using conventional microscopy but the way we manufacture the hydrogels ensures minimal thickness. Additionally thin hydrogels are desirable for immunostaining because thick hydrogels typically introduce high background. Third, our hydrogels are made of two layers, the upper of which is embedded with fluorescent beads, which are confined in a single focal plane ensuring that there is no out of focus light interfering during imaging. That allows the examination of the surface topography of the hydrogels to ensure that the hydrogels are flat and uniform and the performance of 2D or 3D TFM. TFM is a powerful technique that allows the calculation of the traction stresses that cells residing on the hydrogels exert on their matrix (Alvarez-Gonzalez et al., 2015; Steinwachs et al., 2016; del Álamo et al., 2007; Bazellières et al., 2015; Elosegui-Artola et al., 2016). Using our novel assay we have shown that it is possible to calculate the traction stresses of uninfected and infected cells simultaneously and assess the effect of infection in host cell mechanics during the course of infection. In the future one can additionally introduce pharmacological and genetic perturbations to investigate in more depth the complex interplay between host cell mechanics and bacterial infection.

\section{Acknowledgments}

Our thanks to M. Footer, R. Lamason, M. Rengaranjan and members of the Theriot Lab for discussions and experimental support. This work was 
supported by NIH R01AI036929 (J.A.T) and HHMI (J.A.T). Flow cytometry was performed at the Stanford Shared FACS Facility.

\section{Conclusions}

In conclusion, our results provide strong evidence that our novel assay is compatible with the performance of techniques such as flow cytometry, immunostaining and TFM to study in a highly quantitative fashion infection of host cells with bacteria in a much more physiological environment than previously examined. The potential of using our assay to investigate the complex biomechanical interactions of different host cell lines, bacterial pathogens and ECM environments, including protein coatings, might have significant implications in both unraveling the mechanistic strategies by which bacteria infect hosts and in facilitating potential development of therapeutic interventions against infections.

\section{References}

Ahmed, E. M. 2015. Hydrogel: Preparation, characterization, and applications: A review. Journal of Advanced Research, 6(2), pp 105-121.

Alexander, S., Sydow, L. M., Wessels, D. \& Soll, D. R. 1992. Discoidin proteins of Dictyostelium are necessary for normal cytoskeletal organization and cellular morphology during aggregation. Differentiation, 51(3), pp 149-161.

Ali, M. Y., Chuang, C.-Y. \& Saif, M. T. A. 2014. Reprogramming cellular phenotype by soft collagen gels. Soft Matter, 10(44), pp 8829-8837.

Alvarez-Gonzalez, B., Meili, R., Bastounis, E., Firtel, R. A., Lasheras, J. C. \& Del Alamo, J. C. 2015. Three-dimensional balance of cortical tension and axial contractility enables fast amoeboid migration. Biophys $J$, 108(4), pp 821-32.

Bastounis, E., Meili, R., Alonso-Latorre, B., del Álamo, J., Lasheras, J. \& Firtel, R. 2011. The SCAR/WAVE complex is necessary for proper regulation of traction stresses during amoeboid motility. Mol Biol Cell, 21(22), pp 3995-4003.

Bastounis, E., Meili, R., Álvarez-González, B., Francois, J., del Álamo, J. C., Firtel, R. A. \& Lasheras, J. C. 2014. Both contractile axial and lateral traction force dynamics drive amoeboid cell motility. The Journal of Cell Biology, 204(6), pp 1045-1061.

Bazellières, E., Conte, V., Elosegui-Artola, A., Serra-Picamal, X., Bintanel-Morcillo, M., Roca-Cusachs, P., Muñoz, J. J., Sales-Pardo, M., Guimerà, R. \& Trepat, X. 2015. Control of cell-cell forces and collective cell dynamics by the intercellular adhesome. Nat Cell Biol, 17(4), pp 409-420.

Birukova, A. A., Tian, X., Cokic, I., Beckham, Y., Gardel, M. \& Birukov, K. G. 2013. Endothelial barrier disruption and recovery is controlled by substrate stiffness. Microvascular research, 87(50-57).

Chaudhuri, O., Gu, L., Darnell, M., Klumpers, D., Bencherif, S. A., Weaver, J. C., Huebsch, N. \& Mooney, D. J. 2015. Substrate stress relaxation regulates cell spreading. Nat Commun, 6(6364).

Choe, J. E. \& Welch, M. D. 2016. Actin-based motility of bacterial pathogens: mechanistic diversity and its impact on virulence. Pathog Dis. 
Collins, C., Osborne, L. D., Guilluy, C., Chen, Z., O’Brien, E. T., Reader, J. S., Burridge, K., Superfine, R. \& Tzima, E. 2014. Haemodynamic and extracellular matrix cues regulate the mechanical phenotype and stiffness of aortic endothelial cells. Nature Communications, $5(3984$.

Cossart, P. \& Kocks, C. 1994. The actin-based motility of the facultative intracellular pathogen Listeria monocytogenes. Mol Microbiol, 13(3), pp 395-402.

Dabiri, G. A., Sanger, J. M., Portnoy, D. A. \& Southwick, F. S. 1990. Listeria monocytogenes moves rapidly through the host-cell cytoplasm by inducing directional actin assembly. Proceedings of the National Academy of Sciences of the United States of America, 87(16), pp 6068-6072.

del Álamo, J. C., Meili, R., Alonso-Latorre, B., Rodríguez-Rodríguez, J., Aliseda, A., Firtel, R. A. \& Lasheras, J. C. 2007. Spatio-temporal analysis of eukaryotic cell motility by improved force cytometry. Proc. Nat. Acad. Sci., 104(33), pp 133438.

Drevets, D. A., Sawyer, R. T., Potter, T. A. \& Campbell, P. A. 1995. Listeria monocytogenes infects human endothelial cells by two distinct mechanisms. Infect Immun, 63(11), pp 4268-76.

Dussurget, O., Pizarro-Cerda, J. \& Cossart, P. 2004. Molecular determinants of Listeria monocytogenes virulence. Annu Rev Microbiol, 58(587-610.

Edelstein, A. D., Tsuchida, M. A., Amodaj, N., Pinkard, H., Vale, R. D. \& Stuurman, N. 2014. Advanced methods of microscope control using $\mu$ Manager software. Journal of Biological Methods; Vol 1, No 2 (2014).

Elosegui-Artola, A., Oria, R., Chen, Y., Kosmalska, A., Perez-Gonzalez, C., Castro, N., Zhu, C., Trepat, X. \& Roca-Cusachs, P. 2016. Mechanical regulation of a molecular clutch defines force transmission and transduction in response to matrix rigidity. Nat Cell Biol, 18(5), pp 540-548.

Georges, P., Miller, W., Meaney, D., Sawyer, E. \& Janmey, P. A. 2006. Matrices with compliance comparable to that of brain tissue select neuronal over glial growth in mixed cortical cultures. Biophys J, 90(8), pp 3012-3018.

Gouin, E., Gantelet, H., Egile, C., Lasa, I., Ohayon, H., Villiers, V., Gounon, P., Sansonetti, P. J. \& Cossart, P. 1999. A comparative study of the actin-based motilities of the pathogenic bacteria Listeria monocytogenes, Shigella flexneri and Rickettsia conorii. J Cell Sci, 112 ( Pt 11)(1697-708.

Hur, S. S., del Alamo, J. C., Park, J. S., Li, Y. S., Nguyen, H. A., Teng, D., Wang, K. C., Flores, L., Alonso-Latorre, B., Lasheras, J. C. \& Chien, S. 2012. Roles of cell confluency and fluid shear in 3-dimensional intracellular forces in endothelial cells. Proc Natl Acad Sci U S A, 109(28), pp 11110-5.

Ingber, D. E. 2008. Tensegrity-based mechanosensing from macro to micro. Prog. Biophys. Mol. Biol., 97(2-3), pp 163-79.

Jackson, K. A., Iwamoto, M. \& Swerdlow, D. 2010. Pregnancy-associated listeriosis. Epidemiol Infect, 138(10), pp 1503-9.

Kocks, C., Gouin, E., Tabouret, M., Berche, P., Ohayon, H. \& Cossart, P. 1992. L. monocytogenes-induced actin assembly requires the actA gene product, a surface protein. Cell, 68(3), pp 521-31.

Lemichez, E., Lecuit, M., Nassif, X. \& Bourdoulous, S. 2010. Breaking the wall: targeting of the endothelium by pathogenic bacteria. Nat Rev Micro, 8(2), pp 93104.

Mai, J., Sun, C., Li, S. \& Zhang, X. 2007. A microfabricated platform probing cytoskeleton dynamics using multidirectional topographical cues. Biomedical microdevices, 9(4), pp 523-31. 
Mih, J. D., Sharif, A. S., Liu, F., Marinkovic, A., Symer, M. M. \& Tschumperlin, D. J. 2011. A Multiwell Platform for Studying Stiffness-Dependent Cell Biology. PLOS ONE, 6(5), pp e19929.

Minaisah, R.-M., Cox, S. \& Warren, D. T. 2016. The Use of Polyacrylamide Hydrogels to Study the Effects of Matrix Stiffness on Nuclear Envelope Properties. In: Shackleton, S., Collas, P. \& Schirmer, C. E. (eds.) The Nuclear Envelope: Methods and Protocols. New York, NY: Springer New York.

Rajabian, T., Gavicherla, B., Heisig, M., Muller-Altrock, S., Goebel, W., Gray-Owen, S. D. \& Ireton, K. 2009. The bacterial virulence factor InlC perturbs apical cell junctions and promotes cell-to-cell spread of Listeria. Nat Cell Biol, 11(10), pp 1212-8.

Santos, L., Fuhrmann, G., Juenet, M., Amdursky, N., Horejs, C. M., Campagnolo, P. \& Stevens, M. M. 2015. Extracellular Stiffness Modulates the Expression of Functional Proteins and Growth Factors in Endothelial Cells. Adv Healthc Mater.

Selinummi, J., Seppala, J., Yli-Harja, O. \& Puhakka, J. A. 2005. Software for quantification of labeled bacteria from digital microscope images by automated image analysis. Biotechniques, 39(6), pp 859-63.

Steinwachs, J., Metzner, C., Skodzek, K., Lang, N., Thievessen, I., Mark, C., Munster, S., Aifantis, K. E. \& Fabry, B. 2016. Three-dimensional force microscopy of cells in biopolymer networks. Nat Meth, 13(2), pp 171-176.

Theriot, J., Mitchison, T., Tilney, L. \& Portnoy, D. 1992. The rate of actin-based motility of intracellular Listeria monocytogenes equals the rate of actin polymerization. Nature, 357(6375), pp 257-60.

Tilney, L. G. \& Portnoy, D. A. 1989. Actin filaments and the growth, movement, and spread of the intracellular bacterial parasite, Listeria monocytogenes. The Journal of Cell Biology, 109(4), pp 1597-1608.

Tse, J. \& Engler, A. 2011. Stiffness Gradients Mimicking In Vivo Tissue Variation Regulate Mesenchymal Stem Cell Fate. PLoS ONE, 6(1), pp e15978.

Vazquez-Boland, J. A., Kuhn, M., Berche, P., Chakraborty, T., Dominguez-Bernal, G., Goebel, W., Gonzalez-Zorn, B., Wehland, J. \& Kreft, J. 2001. Listeria pathogenesis and molecular virulence determinants. Clin Microbiol Rev, 14(3), pp 584-640.

Vincent, L. G., Choi, Y. S., Alonso-Latorre, B., del Alamo, J. C. \& Engler, A. J. 2013. Mesenchymal stem cell durotaxis depends on substrate stiffness gradient strength. Biotechnol J, 8(4), pp 472-84.

Wilson, S. L. \& Drevets, D. A. 1998. Listeria monocytogenes infection and activation of human brain microvascular endothelial cells. J Infect Dis, 178(6), pp 1658-66.

Yeh, Y.-T., Hur, S. S., Chang, J., Wang, K.-C., Chiu, J.-J., Li, Y.-S. \& Chien, S. 2012. Matrix Stiffness Regulates Endothelial Cell Proliferation through Septin 9. PLoS ONE, 7(10), pp e46889.

Zeldovich, V. B., Robbins, J. R., Kapidzic, M., Lauer, P. \& Bakardjiev, A. I. 2011. Invasive extravillous trophoblasts restrict intracellular growth and spread of Listeria monocytogenes. PLoS Pathog, 7(3), pp e1002005. 